\title{
Permutations and Stratified formulae - a Preservation Theorem
}

\author{
Thomas Forster
}

December 31, 2013

\begin{abstract}
It is shown that the sentences (in the language of Set Theory) preserved by the construction usually used to prove the independence of the axiom of foundation can be characterised syntactically: they are precisely the formulae naturally expressible in the language of simple type theory.
\end{abstract}

The permutation construction that concerns us here is familiar to students of $Z F$ as the technique used in the standard proof of the independence of the axiom of foundation. The technique is originally due to Rieger [1957] and Bernays [1954].

If we have a model $\langle V, \in\rangle$ of $Z F$ and let $\sigma$ be the transposition exchanging the empty set and its singleton. Then we define $x \in_{\sigma} y$ by $x \in \sigma(y)$. It turns out that in the model $V^{\sigma}$ consisting of the old universe and the new membership relation $\epsilon_{\sigma}$ the "old" empty set has become an object identical to its own singleton, and foundation has failed. As it happens all the other axioms of $Z F$ are preserved.

The purpose of this note is to prove a preservation theorem saying that the sentences of the language of set theory preserved by this construction are precisely those that obey a certain typing discipline.

We shall need a few definitions before we can state it, and some more before we can prove it.

\section{DEFINITION}

$\phi$ is a stratified formula of the language of set theory if we can define on the set of variables occurring in $\phi$ a function $\sigma$ into the integers such that if $\phi$ contains an occurrence of ' $x \in y$ ' then $\sigma\left({ }^{\prime} y\right.$ ') $=\sigma\left({ }^{\prime} x\right.$ ') +1 and if $\phi$ contains an occurrence of ' $x=y$ ' then $\sigma\left({ }^{\prime} x\right.$ ' $)=\sigma\left({ }^{\prime} y\right.$ '). If such a function can be defined on the bound variables we say that $\phi$ is weakly stratified.

We are assuming that the language of set theory has no constants: if we wish to prove this preservation theorem for a language with constants we would have to recast the proof with "weakly stratified" for "stratified".

DEFINITION

Let $\mathcal{M}$ be a structure for the language of set theory which is also a model of the axiom of extensionality. Let $\tau$ be a permutation of $\mathcal{M}$. Since $\mathcal{M}$ is a model of some sort of set theory the map $x \mapsto \tau$ " $x$ gives rise to a map defined 
on $\mathcal{M}$, since every element of $\mathcal{M}$ corresponds to a unique subset of $\mathcal{M}$. Let us define $j={ }_{d f} \lambda f \lambda x .\left(f^{\prime \prime} x\right)$ so that this map is the restriction of $j \tau$ to $\mathcal{M}$. It may or may not be onto $\mathcal{M}$, for there is in general no guarantee that that the image of any subset of $\mathcal{M}$ by translation under $\tau$ is a set in the sense of $\mathcal{M}$, which is what we would need for $j \tau$ (or, strictly, its restriction to $\mathcal{M}$ ) to be a permutation of $\mathcal{M}$. This is obviously something to do with the axiom scheme of replacement, and the only assumption we are making about $\mathcal{M}$ is that it is a model of extensionality. If $\tau$ is a permutation of $\mathcal{M}$ such that the restriction of $j^{n} \tau$ to $\mathcal{M}$ is defined on $\mathcal{M}$ and takes values in $M$ then we say that $\tau$ is $n$-setlike. If $j^{n} \tau$ is a permutation of $\mathcal{M}$ for all $n$ then we say that $\tau$ is "setlike". Evidently the setlike permutations form a subgroup of the symmetric group on $\mathcal{M}$.

This definition of 'setlike' is the key to the result, for if $\tau$ is not setlike we do not have enough control over $\mathcal{M}$ (though we remark without proof that if $\tau$ is $n$-setlike then $\mathcal{M}^{\tau}$ will satisfy the same $(n+2)$-stratified formulae as $\mathcal{M}$ (where a formula is $n$-stratified if there is a function assigning integers to its variables that takes at most $n$ distinct values)) and on the other hand if $\tau$ is a set of $\mathcal{M}$ it may preserve some sentences that are not stratified.

\section{DEFINITION}

Let $\left\langle\mathcal{M}, \in_{\mathcal{M}}\right\rangle$ and $\left\langle\mathcal{N}, \in_{\mathcal{N}}\right\rangle$ be two structures for the language of set theory and models of extensionality. Let $\left\langle\pi_{i}: i \in \mathbf{N}\right\rangle$ be a family of maps from $\mathcal{M} \times\{i\}$ to $\mathcal{N} \times\{i\} . \mathcal{M} \times \mathbf{N}$ (" $M^{\infty}$ " for short) has an obvious many sorted structure where for $x$ in $\mathcal{M} \times\{i\}$ and $y$ in $\mathcal{M} \times\{i+1\}$ we say $x \in y$ iff the first component of $x \in \mathcal{M}$ the first component of $y$. Hereafter we shall speak of ' $\mathcal{M}_{i}$ ' instead of ' $\mathcal{M} \times\{i\}$ '. We say $\left\langle\pi_{i}: i \in \mathbf{N}\right\rangle$ is a stratimorphism of $\left\langle\mathcal{M}, \in_{\mathcal{M}}\right\rangle$ and $\left\langle\mathcal{N}, \in_{\mathcal{N}}\right\rangle$ if each $\pi_{i}$ is a bijection and (considering $\pi_{i}$ to be the obvious map from $\mathcal{M}$ to $\mathcal{N}$-we shall make this identification often) we have $\forall x, y \forall i\left(x \in \mathcal{M}_{i} \wedge y \in \mathcal{M}_{i+1} \wedge x \in y\right) \longleftrightarrow \pi_{i}(x) \in \pi_{i+1}(y)$ where the unsubscripted $\in$ is the "obvious" many-sorted relation alluded to above, so that $\pi_{n+1}$ is simply the restriction of $j \pi_{n}$ to $\mathcal{M}$.

\section{THEOREM}

A sentence is equivalent to a stratified sentence iff it is preserved under all permutation models where the permutation is setlike.

(By " $\Phi$ is preserved under permutation models" we mean that any model obtained from a model of $\Phi$ by using permutations in the style is also a model of $\Phi)$.

The proof procedes by a series of lemmas.

LEMMA

If $\left\langle\mathcal{M}, \in_{\mathcal{M}}\right\rangle$ and $\left\langle\mathcal{N}, \in_{\mathcal{N}}\right\rangle$ are stratimorphic then they are elementarily equivalent w.r.t. stratified sentences.

Proof:

If $\left\langle\mathcal{M}, \in_{\mathcal{M}}\right\rangle$ and $\left\langle\mathcal{N}, \in_{\mathcal{N}}\right\rangle$ are stratimorphic that is to say that the two manysorted structures $M^{\infty}$ and $N^{\infty}$ defined above are isomorphic as many-sorted structures: that is precisely the content of the definition of stratimorphism. 
There is a canonical method of interpreting many-sorted theories in one-sorted theories which the reader is assumed to know. I will call it the canonical interpretation. $M^{\infty}$ and $N^{\infty}$ are at least elementarily equivalent with respect to the many-sorted language. But every stratified sentence arises as the value of a sentence in the many-sorted language of typed Set Theory under the canonical interpretation and it is not hard to see that if $\phi$ is a sentence of the many-sorted language then $M^{\infty}$ satifies $\phi$ iff $\left\langle\mathcal{M}, \in_{\mathcal{M}}\right\rangle$ satisfies $\phi$ 's canonical translation. Therefore $\left\langle\mathcal{M}, \in_{\mathcal{M}}\right\rangle$ and $\left\langle\mathcal{N}, \in_{\mathcal{N}}\right\rangle$ satisfy the same stratified sentences.

There is a converse which we shall need. Pétry [1980] has shown:

\section{LEMMA}

If $\left\langle\mathcal{M}, \in_{\mathcal{M}}\right\rangle$ and $\left\langle\mathcal{N}, \in_{\mathcal{N}}\right\rangle$ are elementarily equivalent w.r.t. stratified sentences they have stratimorphic ultrapowers.

Proof:

See Pétry [1980]. Pétry intends to publish a more thorough treatment with some examples in due course. Readers who either have no French or no access to Pétry [1980] may perhaps be able to reconstruct his proof if told that it is like that of the Keisler Ultrapower theorem: use the fact that the ultrapowers are saturated to construct a stratimorphism by a back-and-forth construction.

Next we show that

\section{LEMMA}

If $\left\langle\mathcal{M}, \in_{\mathcal{M}}\right\rangle$ and $\left\langle\mathcal{N}, \in_{\mathcal{N}}\right\rangle$ are two structures for the language of set theory (both models of extensionality) then $\left\langle\mathcal{M}, \in_{\mathcal{M}}\right\rangle$ and $\left\langle\mathcal{N}, \in_{\mathcal{N}}\right\rangle$ are stratimorphic iff $\left\langle\mathcal{M}, \in_{\mathcal{M}}\right\rangle$ is isomorphic to $N^{\tau}$ for some setlike $\tau$.

We prove the two directions in turn:

left to right

If $\left\langle\mathcal{M}, \in_{\mathcal{M}}\right\rangle$ and $\left\langle\mathcal{N}, \in_{\mathcal{N}}\right\rangle$ are stratimorphic then one is a permutation model of the other.

Proof:

$\left\langle\mathcal{M}, \in_{\mathcal{M}}\right\rangle$ and $\left\langle\mathcal{N}, \in_{\mathcal{N}}\right\rangle$ are stratimorphic so let $f_{i}$ be the map $\mathcal{M} \rightarrow \mathcal{N}$ induced by the map sending $\mathcal{M}_{i} 1-1$ onto $\mathcal{N}_{i}$. Therefore for each $n,\left(f_{n+1}\right)^{-1} \circ f_{n}$ is a permutation of $\left\langle\mathcal{M}, \in_{\mathcal{M}}\right\rangle$. Let us call it $\pi_{n}$.

Evidently $\pi_{n+1}$ is the restriction of $j \pi_{n}$ to $\mathcal{M}$, so $\pi_{1}$ is $n$-setlike for each $n$ and therefore setlike. $M^{\pi_{n}}$ (see definition in the first paragraph) is isomorphic to $\left\langle\mathcal{N}, \in_{\mathcal{N}}\right\rangle$ for any $n$ : for instance:

$$
M^{\pi_{1}} \models x \in y
$$

iff

$$
M \models x \in \pi_{1}(y)
$$

iff

$$
\left\langle\mathcal{M}, \in_{\mathcal{M}}\right\rangle \models x \in\left(f_{1}\right)^{-1} \circ f_{0}(y)
$$


iff

$$
N \models f_{0}(x) \in f_{1} \circ\left(f_{1}\right)^{-1} \circ f_{0}(y)=f_{0}(y)
$$

so $f_{0}$ is an isomorphism between $M^{\pi_{1}}$ and $\left\langle\mathcal{N}, \in_{\mathcal{N}}\right\rangle$.

right to left

Let $\left\langle\mathcal{M}, \in_{\mathcal{M}}\right\rangle$ be a structure as above and let $\tau$ be a setlike permutation of $\mathcal{M}$. Then $\left\langle\mathcal{M}, \in_{\mathcal{M}}\right\rangle$ and $M^{\tau}$ are stratimorphic.

Proof:

We will construct $\left\langle\pi_{i}: i \in \mathbf{N}\right\rangle$ by recursion on $i$, where each $\pi_{i}: \mathcal{M} \rightarrow \mathcal{N}$. We turn them into components of a stratimorphism in the obvious way. $\pi_{0}$ is some arbitrary setlike permutation of $\mathcal{M}$, for simplicity's sake the identity. Thereafter we will want to know that

$$
x \in y \longleftrightarrow \pi_{n}(x) \in_{\tau} \pi_{n+1}(y)
$$

which is to say

$$
x \in y \longleftrightarrow \pi_{n}(x) \in \tau \pi_{n+1}(y)
$$

But we have

$$
x \in y \longleftrightarrow \pi_{n}(x) \in\left(j \pi_{n}\right)(y)
$$

since $u \in v \longleftrightarrow \sigma(u) \in(j \sigma)(v)$ for any $u, v$ and any permutation $\sigma$. So by extensionality $\tau \pi_{n+1}(y)=\left(j \pi_{n}\right)(y)$ which is to say

$$
\pi_{n+1}(y)=\left(\tau^{-1}\right)\left(j \pi_{n}\right)(y)
$$

i.e. $\pi_{n+1}=\left(\tau^{-1}\right)\left(j \pi_{n}\right)$ when restricted to $\mathcal{M}$. So for $n \geq 1$ we set $\pi_{n+1}$ to be the restriction of $\left(\tau^{-1}\right)\left(j \pi_{n}\right)$ to $\left.\mathcal{M}\right)$.

This recursive definition of $\pi_{n}$ shows why we have to assume $\tau$ is setlike, for otherwise the output of the recursion will not be defined on the whole of $\mathcal{M}$.

We now know that $\mathcal{M}$ and $\mathcal{N}$ are elementarily equivalent with respect to stratified sentences iff they have stratimorphic ultrapowers and that they are stratimorphic iff they are permutation models of each other. To complete the proof that a sentence is equivalent to a stratified sentence iff it is preserved under permutations we use standard techniques for proving preservation theorems. For example we can use lemma 3.2.1 on page 124 of Chang and Keisler, where $T$ is a theory with one axiom preserved by setlike permutations, and $\Delta$ is the set of all stratified formulae.

One step forward from here would be to prove the following amplification of an earlier remark, here offered without proof - that the result continues to hold if we replace "stratified" by " $(n+2)$-stratified" and "setlike" by " $n$-setlike". In the proof we will need the notion of "stratimorphic with $n$ types" (obtained by replacing " $\in \mathbf{N}$ " by " $<n$ " in the definition of stratimorphism).

However the interesting step is to extend this characterisation of stratified formulae of set theory by means of preservation under permutation to other analogous notions such as "typed" in modern extended $\lambda$-calculi and type theories. A proof of an analogous result for the typed $\lambda$-calculus will follow soon.

Bibliography 
Bernays. P. [1954] A system of axiomatic set theory VII. J.S.L 1954 pp 86-91

Chang C.C., and Keisler H.J. Model Theory. North Holland 1974

Pétry A [1980] Une caractérisation algébrique des structures satisfaisant les mêmes sentences stratifiées. Cahiers du Centre de Logique 4 pp 7-11.

Rieger, L. [1957] A contribution to Gödels axiomatic set theory Czech. Math. J 7 (82) (1957) pp 323-357. 